Review

\title{
Roles of Rho GTPases in Intracellular Transport and Cellular Transformation
}

Xiaojuan Chi ${ }^{1}$, Song Wang ${ }^{2}$, Yifan Huang ${ }^{1}$, Mark Stamnes ${ }^{3}$ and Ji-Long Chen ${ }^{1,2, *}$

1 College of Animal Science, Fujian Agriculture and Forestry University, Fuzhou 350002, China; E-Mails: chixiaojuan88@126.com (X.C.); zjhyfang@163.com (Y.H.)

2 CAS Key Laboratory of Pathogenic Microbiology and Immunology, Institute of Microbiology, Chinese Academy of Sciences (CAS), Beijing 100101, China; E-Mail: wscookie@163.com Department of Molecular Physiology and Biophysics, Roy J. and Lucille A. Carver College of Medicine, The University of Iowa, Iowa City, IA 52242, USA; E-Mail: mark-stamnes@uiowa.edu

* Author to whom correspondence should be addressed; E-Mail: chenj1@im.ac.cn; Tel.: +86-10-6480-7300; Fax: +86-10-6480-7980.

Received: 21 February 2013; in revised form: 4 March 2013 / Accepted: 12 March 2013 / Published: 28 March 2013

\begin{abstract}
Rho family GTPases belong to the Ras GTPase superfamily and transduce intracellular signals known to regulate a variety of cellular processes, including cell polarity, morphogenesis, migration, apoptosis, vesicle trafficking, viral transport and cellular transformation. The three best-characterized Rho family members are Cdc42, RhoA and Rac1. Cdc42 regulates endocytosis, the transport between the endoplasmic reticulum and Golgi apparatus, post-Golgi transport and exocytosis. Cdc42 influences trafficking through interaction with Wiskott-Aldrich syndrome protein (N-WASP) and the Arp2/3 complex, leading to changes in actin dynamics. Rac1 mediates endocytic and exocytic vesicle trafficking by interaction with its effectors, PI3kinase, synaptojanin 2, IQGAP1 and phospholipase D1. RhoA participates in the regulation of endocytosis through controlling its downstream target, Rho kinase. Interestingly, these GTPases play important roles at different stages of viral protein and genome transport in infected host cells. Importantly, dysregulation of $\mathrm{Cdc} 42$, Rac1 and RhoA leads to numerous disorders, including malignant transformation. In some cases, hyperactivation of Rho GTPases is required for cellular transformation. In this article, we review a number of findings related to Rho GTPase function in intracellular transport and cellular transformation.
\end{abstract}


Keywords: Rho GTPases; vesicle trafficking; viral transport; cellular transformation; actin cytoskeleton

\section{Introduction}

The Rho GTPase family, which consists of more than 20 proteins in humans, can be categorized into eight subfamilies according to amino-acid sequence similarities: Rac (Rac1, Rac2, Rac3 and RhoG); Cdc42 (Cdc42, TC10 and TCL); CHP and WRCH1; RhoH; Rho BTB (RhoBTB1, RhoBTB2); Rho (RhoA, RhoB and RhoC); RND (RND1, RND2 and RND3); and Rif (Rif and RhoD) [1]. The members of the four subfamilies (Rac, Cdc42, Rho and Rif) belong to the classical Rho GTPases, while others are parts of the atypical Rho GTPases. Over the past twenty years, Rho GTPases have been implicated in a variety of cellular processes, especially in the regulation of cytoskeletal dynamics [2,3]. Extensive studies have focused on the functional analysis of three important Rho GTPase members: Cdc42, Rac1 and RhoA. Interestingly, recent studies have shown that these Rho GTPases are also involved in cellular trafficking and tumorigenesis.

Like other GTPases, the classical Rho GTPases cycle between active GTP-bound forms and inactive GDP-bound forms. There are three types of regulatory proteins that control the GTPase cycle: guanine nucleotide exchange factors (GEFs), GTPase-activating proteins (GAPs) and guanine nucleotide dissociation inhibitors (GDIs) [4] (Figure 1). GEFs facilitate the release of GDP and the binding of GTP to activate GTPases. GAPs inactivate Rho GTPases by promoting the intrinsic GTP hydrolyzing activity of Rho proteins, resulting in a GDP-bound form. GDIs can bind to $C$-terminal prenyl groups on some Rho proteins to keep the GTPases in the inactive form [5]. Through this GTPase cycle, Rho proteins, together with their GEFs, GAPs and GDI regulators, function as molecular switches in the regulation of a wide range of cellular processes, including cell polarity, cellular adhesion, vesicle trafficking, migration, neuronal development, morphogenesis, proliferation, differentiation and transformation [6-9].

Normal temporal and spatial regulation of vesicular transport events is crucial to cell proliferation and apoptosis, as well as the maintenance of homeostasis. For instance, inhibition of brefeldin A (BFA)-inhibited GEF2 protein (BIG2), which is important for vesicular transport, decreases cell proliferation and disturbs the intracellular localization of E-cadherin and $\beta$-catenin, thereby influencing human cerebral cortical development [10]. Deregulation of vesicular transport can lead to decreased capacitive calcium entry, which in turn results in cell apoptosis [11]. Previous studies have indicated that many Rho GTPases regulate transport pathways, including the endoplasmic reticulum (ER) to the Golgi and endocytic and exocytic transports. In many cases, Rho GTPases play a key role in vesicle trafficking through their ability to regulate the actin cytoskeleton. The Rho GTPases, in an active GTP-bound form, interact with downstream effectors that either directly or indirectly stimulate actin polymerization. Actin plays multiple roles in vesicle trafficking [12-14]. It not only facilitates membrane deformation, cytoskeleton remodeling and the formation of vesicles, but also contributes to vesicle movement and targeting within the cell. 
Figure 1. Rho GTPase activity is controlled by guanine nucleotide exchange factor (GEF), GTPase-activating protein (GAP) and guanine nucleotide dissociation inhibitor (GDI). GEF activates Rho GTPases by facilitating the release of GDP and the binding of GTP. GAP inactivates Rho GTPases by promoting hydrolysis of the bound GTP molecules, resulting in their quick change from the GTP-bound form to the GDP-bound form. GDI binds to $C$-terminal prenyl groups on some Rho proteins, maintaining them in the inactive state. Active Rho GTPases act on their downstream effector proteins, stimulating a variety of cellular processes.

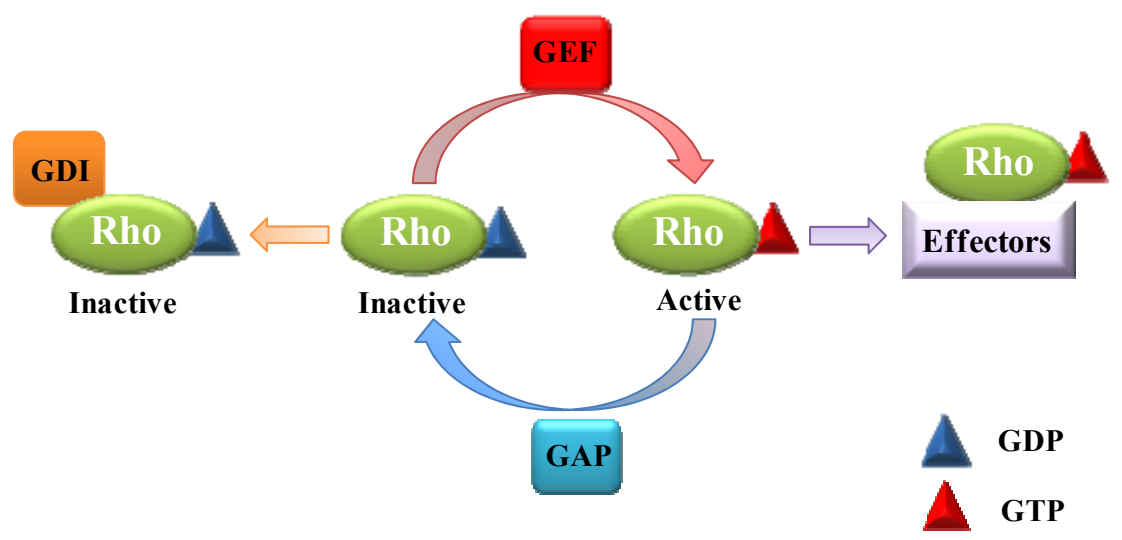

The best-studied Rho family members are Cdc42, Rac1 and RhoA in mammalian cells. Activation of Cdc42 helps control cytoskeletal remodeling, establishment of cell polarity, migration, proliferation and transcription by stimulating a variety of signaling cascades $[15,16]$. In addition, Cdc42 participates in intracellular trafficking and the regulation of malignant transformation, tumor progression and metastasis [17,18]. Rac1 is ubiquitously expressed and involved in signal pathways that regulate mobility and other processes related to membrane trafficking and cell morphology [19,20]. RhoA facilitates the assembly of contractile actomyosin filaments and is associated with focal adhesion complexes. Recently, RhoA has been found to function in vesicle trafficking, tumor invasion and metastasis [21,22].

Other Rho family members, in addition to Cdc42, Rac1 and RhoA, have also been implicated in vesicle trafficking and cell transformation. TC10 is an important molecule for exocytic processes and interacts with one of the members of the exocyst complex, Exo70, which is a major player in exocytosis and has been identified as an effector of $\mathrm{Cdc} 42$ and Rho3 in yeast $[23,24]$. It can regulate the trafficking of glucose transporter 4 (GLUT4) and cystic fibrosis transmembrane conductance regulator (CFTR) [25,26]. Since insulin-mediated activation of TC10 promotes the exocytic processes of GLUT4, it may associate with the development of type 2 diabetes. In human neutrophils, Rac 2 can promote primary granule exocytosis through mediating actin cytoskeletal remodeling $[27,28]$. RhoD is able to link actin reorganization to endosomal vesicle transport [29]. RhoB not only regulates endocytic trafficking, but also acts as a tumor suppressor. Dominant-negative RhoB (RhoBN19 and RhoBV14) affects postendocytic traffic of ligand-receptor complexes from the basolateral endosomes to apical compartments [30]. Furthermore, the activated form of RhoB can delay the endocytic trafficking of the epidermal growth factor receptor (EGFR) [31]. RhoB has also been reported to inhibit tumor growth, cell migration and invasion [32], but the mechanism by which it suppresses 
tumor progression and invasion is still unclear. RhoC has been identified for involvement in tumor metastasis [33]. Rac1b, a tumor associated and constitutively active Rac1 splice variant, is upregulated in colorectal tumor cell and can promote transformation of NIH3T3 cells [34,35]. In this review, we focus on the trafficking and cancer-related functions of Cdc42, Rac1 and RhoA in mammalian cells.

\section{Role of Rho GTPases in Vesicular Transport}

\subsection{Cdc42: A Regulator of Vesicle Trafficking}

Cdc42, a highly conserved small GTPase of the Rho family, was first discovered in the budding yeast Saccharomyces cerevisiae, due to its involvement in the development of cell polarity [36,37]. Since then, Cdc42 has been found to be a molecular switch that modulates a variety of cellular processes in organisms from yeast to mammals, including regulation of actin cytoskeletal architecture and cell polarity, which have been extensively studied. Several studies have implicated Cdc42 in endocytosis, ER/Golgi interface vesicle trafficking, including anterograde (ER-to-Golgi) and retrograde (Golgi-to-ER) transport, post-Golgi transport and exocytosis (Figure 2).

Figure 2. Cdc42, Rac1 and RhoA function in vesicle trafficking. Cdc42 is implicated in endocytosis, trafficking from the cell surface to Golgi region, transport between the endoplasmic reticulum (ER) and Golgi, post-Golgi transport and exocytosis. Rac1 involves in endocytosis and exocytosis, while RhoA mainly takes part in endocytosis.

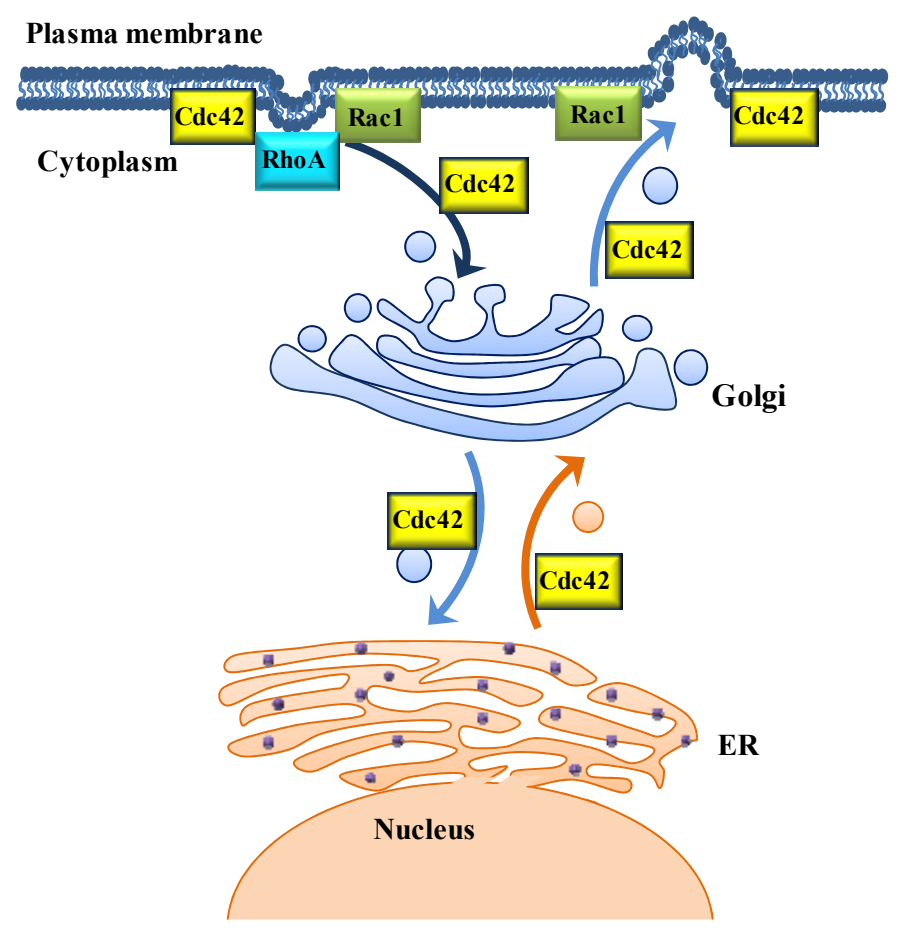

Cdc42 was shown to play a regulatory role in endocytic and secretory pathways, which is important for establishing and maintaining cell polarity [38]. To maintain the cell polarity, some receptors, which express on the apical or basolateral membranes, must be transported to their appropriate location after endocytosis. It has become clear that actin cytoskeleton plays a role in apical and basolateral traffic in 
polarized cells, and Rho GTPases regulate the actin dynamics. Thus, Rho GTPases are involved in maintaining cell polarity. In epithelial cells, Cdc42 regulates polarized endocytosis at the basolateral membrane [39]. Subsequently, Cdc42 has been found to modulate clathrin-dependent endocytosis. Inactivation of Cdc42 by microinjecting a dominant-negative mutant form (Cdc42 T17N) inhibits endocytosis in immature dendritic cells, while the constitutively activate $\mathrm{Cdc} 42$ mutant (Cdc42 Q61L) stimulates endocytosis in mature dendritic cells [40]. Cdc42 is responsible for the uptake of glycosyl-phosphatidylinositol-anchored proteins (GPI-APs) via a clathrin-independent endocytic pathway [41]. Cdc42 helps direct these lipid anchored proteins into the GPI-AP-enriched early endosomal compartment (GEEC). This process is governed by cholesterol-sensitive Cdc42-based actin polymerization $[42,43]$. During endocytosis, Cdc42 interacts with its effector, the Wiskott-Aldrich syndrome protein (N-WASP). N-WASP can then bind and activate the actin-related protein 2/3 (Arp2/3) complex, leading to actin polymerization [44]. Clathrin-mediated endocytosis involves another $\mathrm{Cdc} 42$ effector, called transducer of Cdc42-dependent actin assembly 1 (Toca-1). Toca-1 has three domains, an F-BAR domain, a Cdc42 binding site and a Src homology 3 (SH3) domain. The Cdc42 binding site contributes to the interaction of Toca- 1 and Cdc42 by facilitating the proper folding of Toca-1, while the SH3 domain binds to N-WASP [15]. Forster resonance energy transfer (FRET) experiments have shown that Toca-1, Cdc42 and N-WASP form a trimeric complex on membrane tubules and vesicles. Cdc42 facilitates the assembly of the Toca-1/N-WASP complex by interacting with N-WASP. An important role for this complex may be to induce the formation of membrane tubules and vesicles [45].

Cdc42 has been found to affect several distinct steps in intracellular trafficking. The GTPase-defective mutant (Cdc42 Q61L) and the dominant negative mutant (Cdc42 T17N) both block the transport of glycoprotein of vesicular stomatitis virus (VSV-G) from the ER to the Golgi apparatus. By contrast, the Cdc42 F28L mutant, which binds GTP, accelerates the transport of VSV-G from the ER into the Golgi [46]. In addition, several studies have demonstrated functions for Cdc42 in retrograde trafficking through the secretory pathway. For instance, overexpression and activation of Cdc42 inhibit the retrograde transport of Shiga toxin from the Golgi apparatus to the ER [47]. Furthermore, activation of Cdc42 or knockdown of ARHGAP21 suppresses the retrograde transport of Shiga toxin from the cell periphery to the juxtanuclear Golgi region [48]. Activated Cdc42 also regulates export of bone-specific proteins from the Golgi complex to the cell surface in osteoblasts via the GEF faciogenital dysplasia protein (FGD1) [49], suggesting that Cdc42 also exerts functions in post-Golgi trafficking.

Intracellular membrane trafficking requires the actin cytoskeleton and molecular motor-dependent motility. Because $\mathrm{Cdc} 42$ plays a central role in regulating the actin cytoskeleton dynamics, it is possible that Cdc42 exerts its roles in intracellular trafficking through N-WASP/Arp2/3-regulated actin dynamics [50]. Cdc42 predominantly locates in the Golgi complex in mammalian cells, and it can specifically bind to ARF1-dependent vesicle-coat protein complex coatomer [46,51-53]. The coatomer/Cdc42 complex recruits N-WASP and Arp2/3 to the Golgi membrane. Importantly, p23 (a $\mathrm{COOH}$-terminal dilysine motif on the putative cargo receptor) competes with $\mathrm{Cdc} 42$ for binding to $\gamma \mathrm{COP}$. Once the interaction between Cdc42 and COPI is disrupted by $\mathrm{p} 23$, dynein is recruited to Golgi membranes to promote dynein-dependent vesicle transport. This implies that Cdc42 may help coordinate actin and microtubule-dependent motility events in the secretory pathway. 
Interestingly, the signal pathway of Cdc42/N-WASP/Arp2/3 facilitates protein export by inducing production of actin filaments at the site of exocytosis. A constitutively active Cdc42 mutant can increase exocytosis in chromaffin and PC12 cells [54]. The neuronal guanine nucleotide exchange factor (intersectin-1L), one isoform of the intersectin-1 protein, enhances secretagogue-induced activation of $\mathrm{Cdc} 42$. Activated $\mathrm{Cdc} 42$ recruits N-WASP to the subplasmalemmal region to activate Arp2/3. The increased actin polymerization helps bring granules to the docking sites on the plasma membrane [55].

\subsection{Rac1: Involvement in Endocytic and Exocytic Transport}

Rac1, Ras-related C3 botulinum toxin substrate 1, is the best-characterized member of the Rac subfamily (including Rac1, Rac2, Rac3 and RhoG). Previous studies found that Rac1 regulates a diverse array of cellular events, including the formation of lamellipodia and membrane ruffles, cell cycle, cell adhesion and mobility [21]. In addition, Rac1 regulates endocytic and exocytic trafficking pathways [19] (Figure 2). Unlike Cdc42, Rac1 has not been characterized to participate in the early secretory pathway, nor has it been shown to regulate membrane trafficking at the ER/Golgi interface. Consistent with its function, Rac1 is not located in the Golgi complex [56].

Rac1 has played a characterized role in clathrin-dependent endocytosis [57]. Activation of Rac1, when expressed in intact cells, blocks transferrin-receptor-mediated endocytosis. One mechanism through which Rac1 regulates clathrin-mediated endocytosis is by modulating phosphatidylinositol-3 kinase (PI3K) and synaptojanin 2 (a polyphosphoinositide phosphatase). PI3K, a member of the phosphatidylinositol 4-phosphate 5-kinase family, is an upstream regulator of Rac1. PIP2 is phosphorylated by PI3K to generate PIP3, which induces the activation of Rac1 guanine nucleotide exchange factors (GEFs) to regulate actin rearrangement and vesicular trafficking in various cell types [58]. Recently, P-Rex1, the PI3K-dependent Rac exchange factor, has been identified as a novel regulator of the glucose transporter (GLUT4) trafficking in adipocytes. PIP3 and $\beta \gamma$ subunits of heterotrimeric G-proteins can synergistically activate P-Rex1 [59]. P-Rex1 activates Rac1 to stimulate actin polymerization and facilitate GLUT4 transport to the plasma membrane [60]. Synaptojanin 2, a novel Rac1 effector, can interact with Rac1 directly and specifically [61]. This interaction decreases the formation of coated pits, thereby inhibiting endocytosis.

Recent studies have indicated that activated Rac1 inhibits the endocytosis of E-cadherin through the F-actin cross-linking protein, Ras GTPase-activating-like protein IQGAP1 (IQGAP1), one of its downstream targets. After being activated by trans-interacting E-cadherin, Rac1 can bind to IQGAP1, and then IQGAP1 cross-links F-actin into actin bundles, which inhibits the endocytosis of E-cadherin [62]. Moreover, Rac1 modulates ARF6-mediated clathrin-independent endocytosis through phosphatidydependentinositol-4-phosphate5-kinase (PIP5K). PIP5K binds to the polybasic region (PBR) binding domain of Rac1, whereas calmodulin (CaM) interacts with Rac1 through both the PBR and an adjacent prenyl group. Thus, CaM can disrupt the interaction between Rac1 and PIP5K through steric hindrance. Inhibition of CaM increases the Rac1/PIP5K binding interaction [63]. This interaction is essential for retaining PIP5K at the plasma membrane and for the synthesis of PIP2, which regulates actin dynamics [64]. 
Rac1 has also been reported to regulate calcium-dependent exocytosis in some cells. For instance, a late step of $\mathrm{Ca}^{2+}$-dependent neurotransmitter release is regulated by Rac1 in rat brain synaptosomes [65]. Rac1 can be associated with highly purified synaptic vesicles and is involved in the regulation of neurotransmitter release. Racl activation increases secretion in bovine chromaffin cells [66] and participates in cholecystokinin (CCK)-induced amylase release in pancreatic acinar cells [67]. Early studies indicated that the role of Rac1 in exocytosis is possibly relevant to forming actin-based membrane ruffles [68]. Rac1 redirects the exocytosis of recycling membranes into the sites of ruffle formation. However, the regulatory effects of Rac1 on the calcium-regulated exocytosis might rely on modulating the activity of phospholipase D1 (PLD1). PLD1 plays a major role in late steps of the exocytotic pathway [69]. As an upstream regulator of PLD1, Rac1 could strongly stimulate PLD1 activation and increase the level of phosphatidic acid (PA) at the plasma membrane [70]. The precise mechanisms of Rac1 in exocytosis will require further investigation.

\subsection{RhoA Mainly Participates in the Regulation of Endocytic Transport}

The Rho subfamily of GTPases currently consists of three members: RhoA, RhoB and RhoC. Although these isoforms are highly homologous (RhoA and RhoB share $84 \%$ amino acid sequence identity; RhoA and RhoC share 92\% amino acid sequence identity [1]), they have strikingly different functions in cells. These distinct functions result from $C$-terminal sequence divergence among RhoA, RhoB and RhoC, which affects their localization. RhoA and RhoC are localized in the cytoplasm or at the plasma membrane, whereas RhoB is localized primarily on late endosomes, lysosomes and the plasma membranes. Recently, the functions of these Rho isoforms have extensively studied. RhoA has the remarkable ability to regulate actin polymerization, cell adhesion, actomyosin contractility, cellular transformation, activation of transcription and endocytosis [7,71]. RhoB plays a critical role in cytokine trafficking and cell survival, while RhoC may be able to regulate cell motility [21,72]. Here, we focus on explaining how RhoA regulates endocytosis.

RhoA helps direct endocytosis in a variety of cell types (Figure 2). For example, inactivated RhoA can reduce the uptake of oligomeric $A \beta 42$ in N2A cells as part of the mechanism to defend cells from oligomeric A 342 -induced neurotoxicity [73]. Activated RhoA inhibits transferrin-receptor-mediated endocytosis in Hela cells [57], the muscarinic acetylcholine receptors trafficking to the plasma membrane in HEK293 cells [74] and the uptake of fetuin-A in bovine vascular smooth muscle cells [75]. By contrast, RhoA promotes the endocytosis of interleukin 2 receptors in $L_{\alpha \beta \gamma}$ cells [76] and compensatory endocytosis in umbrella cells [77].

Activated RhoA may affect endocytosis by recruiting the Rho-associated coiled coil-containing protein kinases (ROCKs) to rearrange actin cytoskeleton. The actin cytoskeleton is a highly dynamic structure that promotes the invagination of apical membranes through conjugating myosin motors. As the major downstream effectors of RhoA, ROCKs are involved in two signaling pathways that reorganize the actin cytoskeleton. ROCKs inhibit the myosin light chain phosphatase (MLCP) activity by phosphorylating the myosin binding subunit of MLCP and then increasing the phosphorylation of MLC, thereby enhancing myosin II activity, stress-fiber formation and cellular contraction [78]. Moreover, the ROCK/LIM kinase (LIMK) signaling pathway inactivates cofilin, an actin depolymerization 
factor, resulting in the stabilization of F-actin and the increase of actomyosin contractility [79]. Suppressing the actin-filament severing activity of cofilin leads to stress-fiber formation [80].

Some upstream regulators of RhoA, including PI3K, focal adhesion kinase (FAK), GC-binding factor 2 (GCF2) and GTPase regulator associated with focal adhesion kinase-1 (GRAF1), have been reported to participate in endocytosis and membrane protein trafficking by regulating RhoA activation. Treatment with the PI3K inhibitor (LY294002) or the FAK inhibitor (PF573228) suppresses compensatory endocytosis by $\sim 30 \%$ and $\sim 70 \%$, respectively, by inhibiting the activation of RhoA and then reducing the recruitment of ROCK [77]. Increasing expression of the transcription repressor, GCF2, can silence RhoA expression, leading to actin cytoskeleton disorganization. This increases the internalization of multidrug resistance-associated protein-1 (MRP1) into cytoplasm [81]. GRAF1 participates indirectly in membrane sculpting via regulating RhoA activity. The $N$-terminal BAR and PH domains of GRAF1 localize GRAF1 to membrane tubular structures, [82,83]. Thus, GRAF1 work together with RhoA in directing changes in membrane morphology during endocytosis.

Interestingly, some GAPs and GEFs affect exocytosis by regulating RhoA activity. For instance, GEF-H1, a guanine nucleotide exchange factor for RhoA, can interact with the exocyst component, Sec5. This interaction facilitates RhoA activation, which in turn influences the exocytic trafficking from the Golgi apparatus to the plasma membrane by regulating the assembly and localization of exocyst protein, Exo70 [84]. Gem-interacting protein (GMIP), a RhoA GTPase-activating protein, binds to the secretory factor, JFC1, to orchestrate actin remodeling near secretory organelles by mediating RhoA inactivation and then inducing exocytosis [85].

\section{Rho GTPases Regulate Viral Proteins and Genome Transport in Host Cells}

Given that Cdc42, Rac1 and RhoA are responsible for regulation of host cell protein trafficking, it has been proposed that they play an important role in the intracellular transport of viral proteins and genome. Our previous studies have indicated that Cdc42 and its specific GAP ARHGAP21 are both involved in the transport of influenza A virus neuraminidase (NA) protein. Expression of constitutively active Cdc42 or silencing ARHGAP21 facilitates the transport of NA to the plasma membranes, while expressing shRNA targeting Cdc42 or overexpressing ARHGAP21 significantly decreases the amount of cell surface-localized NA, according to immunofluorescence and NA activity assay [86]. Cdc42 also associates with the influenza A virus matrix protein 1 (M1), and this interaction probably contributes to virus budding [87].

In addition to influenza $\mathrm{A}$ virus, previous studies revealed that $\mathrm{Cdc} 42$, Rac1 and RhoA participate in the lifecycle of multiple viruses. For example, the endocytosis of adenovirus (Ad) and adeno-associated virus type 2 (AAV-2) involves Cdc42 and Rac1 [88-90]. The internalization of Japanese encephalitis virus (JEV) needs the help of RhoA [91]. Cdc42 and Rac1 exert influence early in herpes simplex virus type 1 (HSV-1) infection after the viruses enters the host cells [92]. RhoA is implicated in the trafficking of Kaposi's sarcoma-associated herpes virus (KSHV) DNA to the cell nucleus $[93,94]$. The release of human immunodeficiency virus (HIV-1) and Marburg virus (MARV) particles from infected cells is also strongly influenced by RhoA and Cdc42, respectively [95,96].

The processes of viral endocytosis, intracellular trafficking and exocytosis in the host cells are mostly associated with Rho GTPase-regulated cytoskeletal dynamics. Treatment with CdTB 
(a chemical inhibitor of Rho GTPases) disrupts actin dynamics and prevents the internalization and trafficking of KSHV to the nucleus in human umbilical vein endothelial cells. Wiskostatin (an inhibitor of N-WASP) has similar effects. These suggest that the regulation of actin dynamics through the Rho GTPase /N-WASP/Arp2/3 pathway has an essential role in KSHV entry and trafficking in endothelial cells [97]. In addition, dynamic polymerization of actin in filopodia upon overexpression of Cdc42 promotes the release of MARV particles [96].

Rho GTPases are involved in the regulation of microtubule dynamics and, thus, facilitate the transport of viral pathogens in infected cells and the release of newly assembled virus progeny from host cells. It has been shown that microtubule motors were involved in regulating African swine fever virus (ASFV) entry to host cell and the trafficking of virions to the plasma membrane. Of interest, during the early stages of ASFV infection, activated Rac1 regulates microtubule dynamics by acetylating tubulin, leading to changes in ASFV intracellular transport [98]. RhoA-mDia regulated microtubule dynamics affect viral DNA trafficking in KSHV-infected cells and vaccinia virus release into the media. When infected with KSHV, the RhoA-dependent activation of Dia2 is enhanced in HFF cells [93]. Activated Dia2 directly binds to microtubules and controls their assembly dynamics. This promotes viral DNA transport to the cell nucleus [99]. Besides, dynein motors also play a role in the cytoplasmic trafficking of KSHV and the transport of its DNA to the nucleus [93]. Vaccinia virus F11L protein interacts with RhoA to inhibit the RhoA-ROCK and RhoA-mDia signaling. This is responsible for viral morphogenesis, increased peripheral microtubule dynamics and reorganization of cortical actin, thereby promoting vaccinia virus particle release from infected cells [100-102].

\section{Cdc42, Rac1 and RhoA Contribute to Cellular Transformation and Regulate Tumor Invasion and Metastasis}

Malignant cellular transformation is a complex multifaceted process. Precise spatial and temporal regulation of vesicle trafficking by $\mathrm{Cdc} 42$, Rac1 and RhoA is important for cell survival and function and, hence, contribute to cellular transformation. Perturbation of Cdc42, Rac1 and RhoA activity results in the loss of normal physiological function and can be related to the pathological conditions, including cellular transformation, tumor invasion and metastasis.

The relationship between Cdc42, Rac1 or RhoA and cellular transformation has been extensively studied during the past ten years. This work indicates that hyperactivation of Cdc42, Rac1 or RhoA can lead to cellular transformation. The cycle between GDP- and GTP-bound states of GTPase is critical for the transforming potential of Cdc42, Rac1 and RhoA [103,104]. The spontaneously activated (so-called "fast-cycling") mutants of Cdc42, Rac1 and RhoA that facilitate the intrinsic nucleotide exchange rate, but still exhibit normal GTP hydrolytic activity, can mediate cellular transformation induced by the Dbl oncoprotein in NIH3T3 cells [105]. Cdc42 activation can enhance anchorage-independent cell growth by boosting filopodia formation and generating large, multinucleated cells. RhoA activation results in the loss of contact inhibition and the increase of stress fiber and focal adhesion complexes. Racl activation enhances the accumulation of cortical actin at the cell periphery [105].

Specific GAPs can inhibit the activation of Cdc42, Rac1 and RhoA by increasing intrinsic GTPase activity, leading to suppression of tumor formation. For example, deletion of liver cancer 1 (DLC-1), a 
RhoA and Cdc42-specific GAP, inhibits the growth of various types of cancer, including lung, breast, prostate, kidney, colon, uterus, ovary and stomach through altering the actin cytoskeleton [106,107]. Ankyrin repeat and pleckstrin homology (PH) domain 3 (ARAP3), a specific Rho GAP of Cdc42, Rac 1 and RhoA, has been reported to inhibit peritoneal dissemination of scirrhous gastric carcinoma cells $[106,108]$. The functions of Rho GDIs in cellular transformation are very complex [104] and need further investigation. In some cases, Rho GDIs are thought to inhibit Rho GTPases activation and function as tumor suppressors. Knocking down of RhoGDI1 can promote cancer cell metastasis via inducing RhoA activation [109]. Clinical evidence indicates that RhoGDI2 is a metastasis suppressor in bladder cancer [110]. However, Rho GDIs may not simply inhibit tumor cell metastasis, but also be required for cellular transformation. One study has shown that the interaction between $\mathrm{Cdc} 42$ and RhoGDI is essential for Cdc42-mediated cellular transformation. NIH 3 T3 cells expressing the Cdc42 (F28L, R66A) double mutant, which is ineffective in binding to RhoGDI, fails to proliferate in low (1\%) concentrations of serum and form colonies in soft agar, suggesting that RhoGDI could be involved in cellular transformation [111].

Cdc42, Rac1 and RhoA have the ability to promote cellular transformation, not only due to their own aberrant activation, but also due to their ability to modify oncoproteins, such as Ras, EGFR and sarcoma (Src). Early studies have revealed that Ras-dependent transformation requires functional Cdc42, Rac1 and RhoA [112-115]. These Rho GTPases can impinge on Ras-induced signaling pathways. For example, Cdc42 and Rac1 activate PI3K through indirect cooperative positive feedback [116]. Fibroblast growth factor-2 (FGF-2)-induced activation of Ras/mitogen-activated protein kinase (MAPK) signaling and c-Jun $N$-terminal kinase (JNK) activation require Rac1 activity in human breast cell line MCF7 [117].The p21-activated kinases (PAKs), downstream effectors of $\mathrm{Cdc} 42$, bind to activated $\mathrm{Cdc} 42$ and Rac1 to activate various downstream signaling cascades. This regulates cancer progression by promoting cell proliferation, survival, motility and angiogenesis $[118,119]$. EGFR is often overexpressed or abnormally activated to activate mitogenic signaling pathways in tumor progression. Activation of Cdc42 can prevent EGFR degradation to sustain EGFR signaling and then promote cellular transformation through interacting with c-Cbl, which plays a role in the initiation of EGFR degradation [120]. In addition, Cdc42 may further regulate EGFR-mediated transformation by altering its cellular trafficking. RhoA and ROCK have been shown to negatively regulate EGFR endocytosis. The active form of ROCK can phosphorylate endophilin A1 at Thr-14 and then affect the recruitment of endophilin A1 to the EGFR-c-Cbl-CIN85 complex, leading to the reduction of EGFR endocytosis [121,122]. Similarly, Cdc42, Rac1 and RhoA play roles in cellular transformation mediated by Src [123]. For instance, mDia1, a downstream effector of Rho, is essential for $\mathrm{v}$-Src-induced cellular transformation. Previous studies have shown that v-Src-induced transformation and podosome formation are inhibited in mDial-deficient cells [124]. This can be explained by the fact that mDial deficiency decreases the levels of several tyrosine-phosphorylated proteins, most of which are high molecular mass proteins (100 to $250 \mathrm{kDa}$ ), in v-Src-transformed cells. Subsequently, the translocation of $\mathrm{v}$-Src from the perinuclear region to the cell periphery is impaired, leading to the downregulation of downstream signaling pathways. This ultimately results in the suppression of v-Src-induced cell transformation [124].

Transformed cells exhibit invasive and metastatic properties and the capacity to form invadopodia at the leading edge, which is regulated by actin cytoskeleton reorganization [125]. Cdc42 is thought to 
be important for invadopodia formation, due to induction of actin polymerization via activating the N-WASP/Arp2/3 pathway [126]. N-WASP and the Arp2/3 complex are specifically localized at invadopodia and are essential for invadopodia formation in carcinoma cells. Treatment with N-WASP siRNA or transfection with a N-WASP dominant-negative mutant (lacking the sequence that is required for binding to the Arp2/3 complex) could reduce invadopodia formation in MTLn3 cells [126]. The precise function of N-WASP in invadopodia formation may be related to its ability to promote the assembly of actin filaments through interacting with the Arp2/3 complex or increasing the internalization of degraded matrix components and the recycling of the invadopodia components [127]. In addition, it has been shown that the presence of Rac1 inhibitor, NSC23766, blocks invadopodia formation and matrix degradation [128]. The downstream effector of Rac1, WAVE2, is an important regulator of melanoma invasion and is involved in metastasis. Both enhanced Rac1 activation and increased WAVE2 expression synergistically contribute to B16F10 cell invasion [129]. Moreover, RhoA can also promote tumor cell invasion through regulating invadopodia formation. Inhibition of RhoA activity with $\mathrm{C} 3$ or transfection with dominant-negative RhoA mutant disrupts F-actin accumulation and invadopodia formation [130]. The RhoA effectors, ROCKs and formins, both also have an effect on invadopodia formation. Inhibition of ROCK with specific siRNA reduces invadopodia and decreases tumor cell invasion and knockdown of Dia1, -2 or -3 can inhibit the formation of invadopodia and invasion capacity of MDA-MB-231 cells [131,132]. Taken together, these data suggest that $\mathrm{Cdc} 42$, Rac1 and RhoA are all closely associated with the invasion and metastasis of tumor cells through regulating the formation of invadopodia.

\section{Conclusions}

Because Rho GTPases intimately participate in the regulation of actin cytoskeleton, they play key roles in various physiological and pathological processes, including intracellular transport and tumor progression from transformation to metastasis (Figure 3).

Extensive studies have revealed the pleiotropic effects of Cdc42, Rac1 and RhoA on vesicle trafficking. Although each of them has a specific role, parts of their functions are often overlapping, such as regulating endocytosis. Cdc42 promotes endocytosis through regulation of the Cdc42/Toca-1/N-WASP/Arp2/3 signaling pathway. Rac1 functions in endocytosis by controlling PI3K, synaptojanin 2, IQGAP1 or being involved in Arf6-dependent endocytosis. ROCK plays a crucial role in the RhoA-induced endocytosis. Increasing evidence suggests that there may exist cross talk between the Rho GTPases. However, the precise mechanisms by which signals from these different Rho GTPases are coordinated remain unknown.

In light of previous work, one can conclude that there are cross effects between vesicle trafficking and cellular transformation mediated by $\mathrm{Cdc} 42$, Racl and RhoA. The fact that the two cellular events share the same effectors might explain the phenomenon described above. For example, as a target of Cdc42, COPI can regulate Cdc42-mediated vesicle trafficking and cellular transformation simultaneously [46,51]. Cdc42 mutants that are unable to bind $\gamma$ COP affect both trafficking of VSV-G from the ER to the Golgi apparatus and cellular transformation [46]. The role of ROCK in regulating RhoA-induced endocytosis and cellular transformation has also been documented. Treatment with the ROCK inhibitor, Y-27632, could reduce actin stress fibers, disrupt endocytosis and impair the 
transforming activity of RhoA. However, how Rac1 effectors play roles in both vesicle trafficking and cellular transformation still needs to be determined.

Figure 3. Involvement of $\mathrm{Cdc} 42, \mathrm{Racl}$ and $\mathrm{RhoA}$ in vesicle trafficking, cellular transformation, invadopodia formation and metastasis. Cdc42 regulates vesicle trafficking and invadopodia formation through Wiskott-Aldrich syndrome protein (N-WASP)-mediated Arp2/3 signaling pathway. Rac1 has an effect on endocytosis and exocytosis via acting on its downstream effectors, including synaptojanin 2, IQGAP1, PIP5K and PLD1, and is involved in metastasis through its downstream molecule, WAVE2. RhoA activates its major downstream effector, Rho-associated coiled coil-containing protein kinases (ROCK), to modulate endocytosis and invadopodia formation through affecting actin dynamics. Hyperactivation of Cdc42, Rac1 or RhoA can result in cellular transformation.

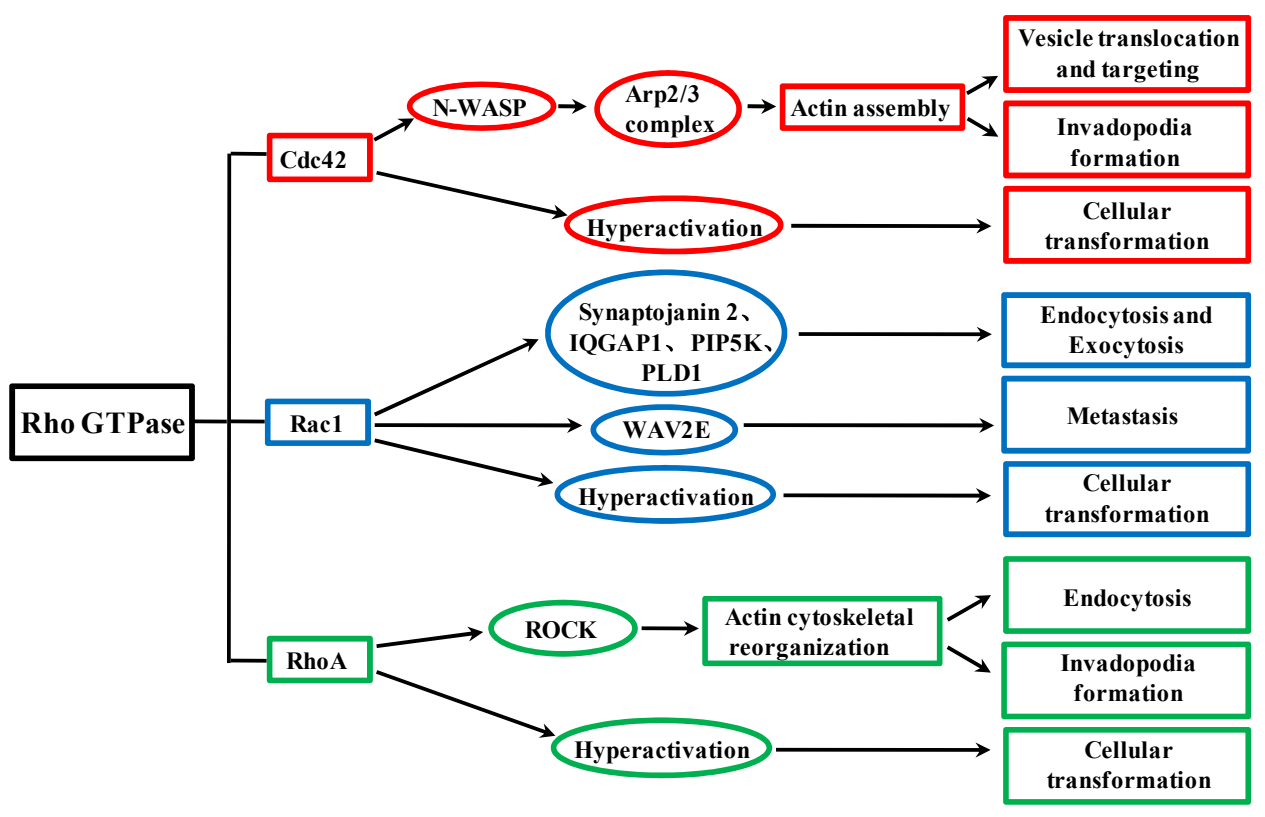

This review focuses mostly on Rho family hallmarks members (Cdc42, Rac1 and RhoA), but some of other members are also closely associated with intracellular transport and tumorigenesis. In the future, more precise investigation of the regulatory roles of the "classical" and "nonclassical" members of the Rho GTPases family will yield additional fascinating insights.

\section{Acknowledgments}

We thank members of the Chen laboratory for helpful discussions. This work was supported by the National Basic Research Program (973) of China (2009CB918902), the National Key Technologies Research and Development Program of China (2013ZX10004-611) and the Hundreds of Talents Program of the Chinese Academy of Sciences (2009-2014) to J.-L. Chen.

\section{Conflict of Interest}

The authors declare no conflict of interest. 


\section{References}

1. Heasman, S.J.; Ridley, A.J. Mammalian Rho GTPases: New insights into their functions from in vivo studies. Nat. Rev. Mol. Cell Biol. 2008, 9, 690-701.

2. Hall, A. Rho GTPases and the actin cytoskeleton. Science 1998, 279, 509-514.

3. Tapon, N.; Hall, A. Rho, Rac and Cdc42 GTPases regulate the organization of the actin cytoskeleton. Curr. Opin. Cell Biol. 1997, 9, 86-92.

4. Boguski, M.S.; McCormick, F. Proteins regulating Ras and its relatives. Nature 1993, 366, 643-654.

5. Symons, M.; Settleman, J. Rho family GTPases: More than simple switches. Trends Cell Biol. 2000, 10, 415-419.

6. Jaffe, A.B.; Hall, A. Rho GTPases: Biochemistry and biology. Annu. Rev. Cell Dev. Biol. 2005, 21, 247-269.

7. Symons, M.; Rusk, N. Control of vesicular trafficking by Rho GTPases. Curr. Biol. 2003, 13, 409-418.

8. Wu, H.; Rossi, G.; Brennwald, P. The ghost in the machine: small GTPases as spatial regulators of exocytosis. Trends Cell Biol. 2008, 18, 397-404.

9. Delprato, A. Topological and functional properties of the small GTPases protein interaction network. PLoS One 2012, 7, e44882.

10. Sheen, V.L.; Ganesh, V.S.; Topcu, M.; Sebire, G.; Bodell, A.; Hill, R.S.; Grant, P.E.; Shugart, Y.Y.; Imitola, J.; Khoury, S.J. Mutations in ARFGEF2 implicate vesicle trafficking in neural progenitor proliferation and migration in the human cerebral cortex. Nat. Genet. 2003, 36, 69-76.

11. Jayadev, S.; Petranka, J.G.; Cheran, S.K.; Biermann, J.A.; Barrett, J.C.; Murphy, E. Reduced capacitative calcium entry correlates with vesicle accumulation and apoptosis. J. Biol. Chem. 1999, 274, 8261-8268.

12. Egea, G.; Lázaro-Diéguez, F.; Vilella, M. Actin dynamics at the Golgi complex in mammalian cells. Curr. Opin. Cell Biol. 2006, 18, 168-178.

13. Smythe, E.; Ayscough, K.R. Actin regulation in endocytosis. J. Cell Sci. 2006, 119, 4589-4598.

14. Lanzetti, L. Actin in membrane trafficking. Curr. Opin. Cell Biol. 2007, 19, 453-458.

15. Ho, H.Y.H.; Rohatgi, R.; Lebensohn, A.M.; Li, J.; Gygi, S.P.; Kirschner, M.W. Toca-1 mediates Cdc42-dependent actin nucleation by activating the N-WASP-WIP complex. Cell 2004, 118, 203-216.

16. Ridley, A.J.; Schwartz, M.A.; Burridge, K.; Firtel, R.A.; Ginsberg, M.H.; Borisy, G.; Parsons, J.T.; Horwitz, A.R. Cell migration: integrating signals from front to back. Science 2003, 302, 1704-1709.

17. Cerione, R.A. Cdc42: New roads to travel. Trends Cell Biol. 2004, 14, 127-132.

18. Gupta, G.P.; Massagué, J. Cancer metastasis: Building a framework. Cell 2006, 127, 679-695.

19. Ridley, A.J. Rho proteins: linking signaling with membrane trafficking. Traffic 2001, 2, 303-310.

20. Vega, F.M.; Ridley, A.J. Rho GTPases in cancer cell biology. FEBS Lett. 2008, 582, 2093-2101. 
21. Ridley, A.J. Rho GTPases and actin dynamics in membrane protrusions and vesicle trafficking. Trends Cell Biol. 2006, 16, 522-529.

22. Wu, D.; Asiedu, M.; Wei, Q. MyoGEF regulates the invasion activity of MDA-MB-231 breast cancer cells through activation of RhoA and RhoC. Oncogene 2009, 28, 2219-2230.

23. Wu, H.; Brennwald, P. The function of two Rho family GTPases is determined by distinct patterns of cell surface localization. Mol. Cell. Biol. 2010, 30, 5207-5217.

24. Adamo, J.E.; Rossi, G.; Brennwald, P. The Rho GTPase Rho3 has a direct role in exocytosis that is distinct from its role in actin polarity. Mol. Biol. Cell. 1999, 10, 4121-4133.

25. Chiang, S.H.; Baumann, C.A.; Kanzaki, M.; Thurmond, D.C.; Watson, R.T.; Neudauer, C.L.; Macara, I.G.; Pessin, J.E.; Saltiel, A.R. Insulin-stimulated GLUT4 translocation requires the CAP-dependent activation of TC10. Nature 2001, 410, 944-948.

26. Cheng, J.; Wang, H.; Guggino, W.B. Regulation of cystic fibrosis transmembrane regulator trafficking and protein expression by a Rho family small GTPase TC10. J. Biol. Chem. 2005, 280, 3731-3739.

27. Mitchell, T.; Lo, A.; Logan, M.R.; Lacy, P.; Eitzen, G. Primary granule exocytosis in human neutrophils is regulated by Rac-dependent actin remodeling. Am. J. Physiol. Cell Physiol. 2008, $295,1354-1365$.

28. Abdel-Latif, D.; Steward, M.; Macdonald, D.L.; Francis, G.A.; Dinauer, M.C.; Lacy, P. Rac2 is critical for neutrophil primary granule exocytosis. Blood 2004, 104, 832-839.

29. Gad, A.K.B.; Aspenström, P. Rif proteins take to the RhoD: Rho GTPases at the crossroads of actin dynamics and membrane trafficking. Cell. Signal. 2010, 22, 183-189.

30. Rondanino, C.; Rojas, R.; Ruiz, W.G.; Wang, E.; Hughey, R.P.; Dunn, K.W.; Apodaca, G. RhoB-dependent modulation of postendocytic traffic in polarized Madin-Darby canine kidney cells. Traffic 2007, 8, 932-949.

31. Wherlock, M.; Gampel, A.; Futter, C.; Mellor, H. Farnesyltransferase inhibitors disrupt EGF receptor traffic through modulation of the RhoB GTPase. J. Cell Sci. 2004, 117, 3221-3231.

32. Huang, M.; Prendergast, G. RhoB in cancer suppression. Histol. Histopathol. 2006, 21, 213-218.

33. Iiizumi, M.; Bandyopadhyay, S.; Pai, S.K.; Watabe, M.; Hirota, S.; Hosobe, S.; Tsukada, T.; Miura, K.; Saito, K.; Furuta, E. RhoC promotes metastasis via activation of the Pyk2 pathway in prostate cancer. Cancer Res. 2008, 68, 7613-7620.

34. Matos, P.; Jordan, P. Increased Rac1b expression sustains colorectal tumor cell survival. Mol. Cancer Res. 2008, 6, 1178-1184.

35. Singh, A.; Karnoub, A.E.; Palmby, T.R.; Lengyel, E.; Sondek, J.; Der, C.J. Raclb, a tumor associated, constitutively active Rac1 splice variant, promotes cellular transformation. Oncogene 2004, 23, 9369-9380.

36. Hartwell, L.H.; Culotti, J.; Pringle, J.R.; Reid, B.J. Genetic control of the cell division cycle in yeast. Science 1974, 183, 46-51.

37. Johnson, D.I.; Pringle, J.R. Molecular characterization of CDC42, a Saccharomyces cerevisiae gene involved in the development of cell polarity. J. Cell Biol. 1990, 111, 143-152.

38. Kroschewski, R.; Hall, A.; Mellman, I. Cdc42 controls secretory and endocytic transport to the basolateral plasma membrane of MDCK cells. Nat. Cell Biol. 1999, 1, 8-13. 
39. Ellis, S.; Mellor, H. Regulation of endocytic traffic by Rho family GTPases. Trends Cell Biol. 2000, $10,85-88$.

40. Garrett, W.S.; Chen, L.M.; Kroschewski, R.; Ebersold, M.; Turley, S.; Trombetta, S.; Galán, J.E.; Mellman, I. Developmental control of endocytosis in dendritic cells by Cdc42. Cell 2000, 102, 325-334.

41. Sabharanjak, S.; Sharma, P.; Parton, R.G.; Mayor, S. GPI-anchored proteins are delivered to recycling endosomes via a distinct cdc42-regulated, clathrin-independent pinocytic pathway. Dev. Cell 2002, 2, 411-423.

42. Chadda, R.; Howes, M.T.; Plowman, S.J.; Hancock, J.F.; Parton, R.G.; Mayor, S. Cholesterol-sensitive $\mathrm{Cdc} 42$ activation regulates actin polymerization for endocytosis via the GEEC pathway. Traffic 2007, 8, 702-717.

43. Mayor, S.; Pagano, R.E. Pathways of clathrin-independent endocytosis. Nat. Rev. Mol. Cell Biol. 2007, 8, 603-612.

44. Cory, G.O.C.; Cramer, R.; Blanchoin, L.; Ridley, A.J. Phosphorylation of the WASP-VCA domain increases its affinity for the Arp $2 / 3$ complex and enhances actin polymerization by WASP. Mol. Cell 2003, 11, 1229-1239.

45. Bu, W.; Lim, K.B.; Yu, Y.H.; Chou, A.M.; Sudhaharan, T.; Ahmed, S. Cdc42 interaction with N-WASP and Toca-1 regulates membrane tubulation, vesicle formation and vesicle motility: implications for endocytosis. PLoS One 2010, 5, e12153.

46. Wu, W.J.; Erickson, J.W.; Lin, R.; Cerione, R.A. The $\gamma$-subunit of the coatomer complex binds Cdc42 to mediate transformation. Nature 2000, 405, 800-804.

47. Luna, A.; Matas, O.B.; Martínez-Menárguez, J.A.; Mato, E.; Durán, J.M.; Ballesta, J.; Way, M.; Egea, G. Regulation of protein transport from the Golgi complex to the endoplasmic reticulum by CDC42 and N-WASP. Mol. Biol. Cell. 2002, 13, 866-879.

48. Hehnly, H.; Longhini, K.M.; Chen, J.L.; Stamnes, M. Retrograde Shiga toxin trafficking is regulated by ARHGAP21 and Cdc42. Mol. Biol. Cell. 2009, 20, 4303-4312.

49. Egorov, M.V.; Capestrano, M.; Vorontsova, O.A.; di Pentima, A.; Egorova, A.V.; Mariggiò, S.; Ayala, M.I.; Tetè, S.; Gorski, J.L.; Luini, A. Faciogenital dysplasia protein (FGD1) regulates export of cargo proteins from the golgi complex via Cdc42 activation. Mol. Biol. Cell. 2009, 20, 2413-2427.

50. Rohatgi, R.; Ma, L.; Miki, H.; Lopez, M.; Kirchhausen, T.; Takenawa, T.; Kirschner, M.W. The interaction between N-WASP and the Arp2/3 complex links Cdc42-dependent signals to actin assembly. Cell 1999, 97, 221-231.

51. Chen, J.L.; Fucini, R.V.; Lacomis, L.; Erdjument-Bromage, H.; Tempst, P.; Stamnes, M. Coatomer-bound Cdc42 regulates dynein recruitment to COPI vesicles. J. Cell Biol. 2005, 169, 383-389.

52. Chen, J.L.; Lacomis, L.; Erdjument-Bromage, H.; Tempst, P.; Stamnes, M. Cytosol-derived proteins are sufficient for Arp2/3 recruitment and ARF/coatomer-dependent actin polymerization on Golgi membranes. FEBS Lett. 2004, 566, 281-286.

53. Harris, K.P.; Tepass, U. Cdc42 and vesicle trafficking in polarized cells. Traffic 2010, 11, $1272-1279$. 
54. Malacombe, M.; Ceridono, M.; Calco, V.; Chasserot-Golaz, S.; McPherson, P.S.; Bader, M.F.; Gasman, S. Intersectin-1L nucleotide exchange factor regulates secretory granule exocytosis by activating Cdc42. EMBO J. 2006, 25, 3494-3503.

55. Gasman, S.; Chasserot-Golaz, S.; Malacombe, M.; Way, M.; Bader, M.F. Regulated exocytosis in neuroendocrine cells: A role for subplasmalemmal Cdc42/N-WASP-induced actin filaments. Mol. Biol. Cell. 2004, 15, 520-531.

56. Matas, O.B.; Fritz, S.; Luna, A.; Egea, G. Membrane trafficking at the ER/Golgi interface: Functional implications of RhoA and Rac1. Eur. J. Cell Biol. 2005, 84, 699-707.

57. Lamaze, C.; Chuang, T.H.; Terlecky, L.J.; Bokoch, G.M.; Schmid, S.L. Regulation of receptor-mediated endocytosis by Rho and Rac. Nature 1996, 382, 177-179.

58. Oikawa, T.; Yamaguchi, H.; Itoh, T.; Kato, M.; Ijuin, T.; Yamazaki, D.; Suetsugu, S.; Takenawa, T. PtdIns (3, 4, 5) P3 binding is necessary for WAVE2-induced formation of lamellipodia. Nat. Cell Biol. 2004, 6, 420-426.

59. Wertheimer, E.; Gutierrez-Uzquiza, A.; Rosemblit, C.; Lopez-Haber, C.; Sosa, M.S.; Kazanietz, M.G. Rac signaling in breast cancer: A tale of GEFs and GAPs. Cell. Signal. 2012, 24, 353-362.

60. Balamatsias, D.; Kong, A.M.; Waters, J.E.; Sriratana, A.; Gurung, R.; Bailey, C.G.; Rasko, J.E.J.; Tiganis, T.; Macaulay, S.L.; Mitchell, C.A. Identification of P-Rex1 as a novel Rac1-guanine nucleotide exchange factor (GEF) that promotes actin remodeling and GLUT4 protein trafficking in adipocytes. J. Biol. Chem. 2011, 286, 43229-43240.

61. Malecz, N.; McCabe, P.C.; Spaargaren, C.; Qiu, R.G.; Chuang, Y.; Symons, M. Synaptojanin 2, a novel Rac1 effector that regulates clathrin-mediated endocytosis. Curr. Biol. 2000, 10, 1383-1386.

62. Izumi, G.; Sakisaka, T.; Baba, T.; Tanaka, S.; Morimoto, K.; Takai, Y. Endocytosis of E-cadherin regulated by Rac and Cdc42 small G proteins through IQGAP1 and actin filaments. J. Cell Biol. 2004, 166, 237-248.

63. Vidal-Quadras, M.; Gelabert-Baldrich, M.; Soriano-Castell, D.; Lladó, A.; Rentero, C.; Calvo, M.; Pol, A.; Enrich, C.; Tebar, F. Rac1 and calmodulin interactions modulate dynamics of ARF6-dependent endocytosis. Traffic 2011, 12, 1879-1896.

64. Van den Bout, I.; Divecha, N. PIP5K-driven PtdIns $(4,5)$ P2 synthesis: regulation and cellular functions. J. Cell Sci. 2009, 122, 3837-3850.

65. Doussau, F.; Gasman, S.; Humeau, Y.; Vitiello, F.; Popoff, M.; Boquet, P.; Bader, M.F.; Poulain, B. A Rho-related GTPase is involved in $\mathrm{Ca}^{2+}$-dependent neurotransmitter exocytosis. J. Biol. Chem. 2000, 275, 7764-7770.

66. Li, Q.; Ho, C.S.; Marinescu, V.; Bhatti, H.; Bokoch, G.M.; Ernst, S.A.; Holz, R.W.; Stuenkel, E.L. Facilitation of $\mathrm{Ca}^{2+}$-dependent exocytosis by Rac1-GTPase in bovine chromaffin cells. J. Physiol. 2004, 550, 431-445.

67. Bi, Y.; Williams, J.A. A role for Rho and Rac in secretagogue-induced amylase release by pancreatic acini. Am. J. Physiol. Cell Physiol. 2005, 289, 22-32.

68. Bretscher, M.S.; Aguado-Velasco, C. EGF induces recycling membrane to form ruffles. Curr. Biol. 1998, 8, 721-724.

69. Momboisse, F.; Ory, S.; Ceridono, M.; Calco, V.; Vitale, N.; Bader, M.F.; Gasman, S. The Rho guanine nucleotide exchange factors intersectin $1 \mathrm{~L}$ and $\beta$-Pix control calcium-regulated exocytosis in neuroendocrine PC12 cells. Cell. Mol. Neurobiol. 2010, 30, 1327-1333. 
70. Momboisse, F.; Lonchamp, E.; Calco, V.; Ceridono, M.; Vitale, N.; Bader, M.F.; Gasman, S. beta PIX-activated Racl stimulates the activation of phospholipase D, which is associated with exocytosis in neuroendocrine cells. J. Cell Sci. 2009, 122, 798-806.

71. Chen, Y.; Yang, Z.; Meng, M.; Zhao, Y.; Dong, N.; Yan, H.; Liu, L.; Ding, M.; Peng, H.B.; Shao, F. Cullin mediates degradation of RhoA through evolutionarily conserved BTB adaptors to control actin cytoskeleton structure and cell movement. Mol. Cell 2009, 35, 841-855.

72. Wheeler, A.P.; Ridley, A.J. Why three Rho proteins? RhoA, RhoB, RhoC, and cell motility. Exp. Cell Res. 2004, 301, 43-49.

73. Yu, C.; Nwabuisi-Heath, E.; Laxton, K.; LaDu, M.J. Endocytic pathways mediating oligomeric A $\beta 42$ neurotoxicity. Mol. Neurodegener. 2010, 5, 19.

74. Vögler, O.; Krummenerl, P.; Schmidt, M.; Jakobs, K.H.; Van Koppen, C.J. RhoA-sensitive trafficking of muscarinic acetylcholine receptors. J. Pharmacol. Exp. Ther. 1999, 288, 36-42.

75. Chen, N.X.; Chen, X.; O’Neill, K.D.; Atkinson, S.J.; Moe, S.M. RhoA/Rho kinase (ROCK) alters fetuin-A uptake and regulates calcification in bovine vascular smooth muscle cells (BVSMC). Am. J. Physiol. Renal. Physiol. 2010, 299, 674-680.

76. Lamaze, C.; Dujeancourt, A.; Baba, T.; Lo, C.G.; Benmerah, A.; Dautry-Varsat, A. Interleukin 2 receptors and detergent-resistant membrane domains define a clathrin-independent endocytic pathway. Mol. Cell 2001, 7, 661-671.

77. Khandelwal, P.; Ruiz, W.G.; Apodaca, G. Compensatory endocytosis in bladder umbrella cells occurs through an integrin-regulated and RhoA-and dynamin-dependent pathway. EMBO J. 2010, 29, 1961-1975.

78. Riento, K.; Ridley, A.J. Rocks: multifunctional kinases in cell behaviour. Nat. Rev. Mol. Cell Biol. 2003, 4, 446-456.

79. Maekawa, M.; Ishizaki, T.; Boku, S.; Watanabe, N.; Fujita, A.; Iwamatsu, A.; Obinata, T.; Ohashi, K.; Mizuno, K.; Narumiya, S. Signaling from Rho to the actin cytoskeleton through protein kinases ROCK and LIM-kinase. Science 1999, 285, 895-898.

80. Bishop, A.L.; Hall, A. Rho GTPases and their effector proteins. Biochem. J. 2000, 348, 241-255.

81. Shen, D.W.; Pouliot, L.M.; Gillet, J.P.; Ma, W.; Johnson, A.C.; Hall, M.D.; Gottesman, M.M. The transcription factor GCF2 is an upstream repressor of the small GTPase RhoA, regulating membrane protein trafficking, sensitivity to doxorubicin, and resistance to cisplatin. Mol. Pharm. 2012, 9, 1822-1833.

82. Lundmark, R.; Doherty, G.J.; Howes, M.T.; Cortese, K.; Vallis, Y.; Parton, R.G.; McMahon, H.T. The GTPase-activating protein GRAF1 regulates the CLIC/GEEC endocytic pathway. Curr. Biol. 2008, 18, 1802-1808.

83. Doherty, G.J.; Lundmark, R. GRAF1-dependent endocytosis. Biochem. Soc. Trans. 2009, 37, 1061-1065.

84. Pathak, R.; Delorme-Walker, V.D.; Howell, M.C.; Anselmo, A.N.; White, M.A.; Bokoch, G.M.; DerMardirossian, C. The microtubule-associated Rho activating factor GEF-H1 interacts with exocyst complex to regulate vesicle traffic. Dev. Cell 2012, 23, 397-411.

85. Johnson, J.L.; Monfregola, J.; Napolitano, G.; Kiosses, W.B.; Catz, S.D. Vesicular trafficking through cortical actin during exocytosis is regulated by the Rab27a effector JFC1/Slp1 and the RhoA-GTPase-activating protein Gem-interacting protein. Mol. Biol. Cell. 2012, 23, 1902-1916. 
86. Wang, S.; Li, H.; Chen, Y.; Wei, H.; Gao, G.F.; Liu, H.; Huang, S.; Chen, J.L. Transport of influenza virus neuraminidase (NA) to host cell surface is regulated by ARHGAP21 and Cdc42 proteins. J. Biol. Chem. 2012, 287, 9804-9816.

87. Hui, E.K.W.; Barman, S.; Tang, D.H.P.; France, B.; Nayak, D.P. YRKL sequence of influenza virus M1 functions as the L domain motif and interacts with VPS28 and Cdc42. J. Virol. 2006, 80, 2291-2308.

88. Li, E.; Stupack, D.; Bokoch, G.M.; Nemerow, G. R. Adenovirus endocytosis requires actin cytoskeleton reorganization mediated by Rho family GTPases. J. Virol. 1998, 72, 8806-8812.

89. Sanlioglu, S.; Benson, P.K.; Yang, J.; Atkinson, E.M.; Reynolds, T.; Engelhardt, J.F. Endocytosis and nuclear trafficking of adeno-associated virus type 2 are controlled by Rac1 and phosphatidylinositol-3 kinase activation. J. Virol. 2000, 74, 9184-9196.

90. Nonnenmacher, M.; Weber, T. Adeno-associated virus 2 infection requires endocytosis through the CLIC/GEEC pathway. Cell Host Microbe 2011, 10, 563-576.

91. Kalia, M.; Khasa, R.; Sharma, M.; Nain, M.; Vrati, S. Japanese encephalitis virus infects neuronal cells through a clathrin-independent endocytic mechanism. J. Virol. 2013, 87, 148-162.

92. Hoppe, S.; Schelhaas, M.; Jaeger, V.; Liebig, T.; Petermann, P.; Knebel-Mörsdorf, D. Early herpes simplex virus type 1 infection is dependent on regulated Rac1/Cdc42 signalling in epithelial MDCKII cells. J. Gen. Virol. 2006, 87, 3483-3494.

93. Naranatt, P.P.; Krishnan, H.H.; Smith, M.S.; Chandran, B. Kaposi's sarcoma-associated herpesvirus modulates microtubule dynamics via RhoA-GTP-diaphanous 2 signaling and utilizes the dynein motors to deliver its DNA to the nucleus. J. Virol. 2005, 79, 1191-1206.

94. Raghu, H.; Sharma-Walia, N.; Veettil, M.V.; Sadagopan, S.; Caballero, A.; Sivakumar, R.; Varga, L.; Bottero, V.; Chandran, B. Lipid rafts of primary endothelial cells are essential for Kaposi's sarcoma-associated herpesvirus/human herpesvirus 8-induced phosphatidylinositol 3-kinase and RhoA-GTPases critical for microtubule dynamics and nuclear delivery of viral DNA but dispensable for binding and entry. J. Virol. 2007, 81, 7941-7959.

95. Loomis, R.J.; Holmes, D.A.; Elms, A.; Solski, P.A.; Der, C.J.; Su, L. Citron kinase, a RhoA effector, enhances HIV-1 virion production by modulating exocytosis. Traffic 2006, 7, 1643-1653.

96. Kolesnikova, L.; Bohil, A.B.; Cheney, R.E.; Becker, S. Budding of Marburgvirus is associated with filopodia. Cell. Microbiol. 2006, 9, 939-951.

97. Greene, W.; Gao, S.J. Actin dynamics regulate multiple endosomal steps during Kaposi's sarcoma-associated herpesvirus entry and trafficking in endothelial cells. PLoS Pathog. 2009, 5, e1000512.

98. Quetglas, J.I.; Hernáez, B.; Galindo, I.; Muñoz-Moreno, R.; Cuesta-Geijo, M.A.; Alonso, C. Small Rho GTPases and cholesterol biosynthetic pathway intermediates in African swine fever virus infection. J. Virol. 2012, 86, 1758-1767.

99. Palazzo, A.F.; Cook, T.A.; Alberts, A.S.; Gundersen, G.G. mDia mediates Rho-regulated formation and orientation of stable microtubules. Nat. Cell Biol. 2001, 3, 723-729.

100. Arakawa, Y.; Cordeiro, J.V.; Way, M. F11L-mediated inhibition of RhoA-mDia signaling stimulates microtubule dynamics during vaccinia virus infection. Cell Host Microbe 2007, 1, 213-226. 
101. Arakawa, Y.; Cordeiro, J.V.; Schleich, S.; Newsome, T.P.; Way, M. The release of vaccinia virus from infected cells requires RhoA-mDia modulation of cortical actin. Cell Host Microbe 2007, 1, 227-240.

102. Valderrama, F.; Cordeiro, J.V.; Schleich, S.; Frischknecht, F.; Way, M. Vaccinia virus-induced cell motility requires F11L-mediated inhibition of RhoA signaling. Science 2006, 311, 377-381.

103. Bos, J.L.; Rehmann, H.; Wittinghofer, A. GEFs and GAPs: Critical elements in the control of small G proteins. Cell 2007, 129, 865-877.

104. Stengel, K.; Zheng, Y. Cdc42 in oncogenic transformation, invasion, and tumorigenesis. Cell. Signal. 2011, 23, 1415-1423.

105. Lin, R.; Cerione, R.A.; Manor, D. Specific contributions of the small GTPases Rho, Rac, and Cdc42 to Dbl transformation. J. Biol. Chem. 1999, 274, 23633-23641.

106. Tcherkezian, J.; Lamarche-Vane, N. Current knowledge of the large RhoGAP family of proteins. Biol. Cell 2007, 99, 67-86.

107. Liao, Y.C.; Lo, S.H. Deleted in liver cancer-1 (DLC-1): A tumor suppressor not just for liver. Int. J. Biochem. Cell Biol. 2008, 40, 843-847.

108. Yagi, R.; Tanaka, M.; Sasaki, K.; Kamata, R.; Nakanishi, Y.; Kanai, Y.; Sakai, R. ARAP3 inhibits peritoneal dissemination of scirrhous gastric carcinoma cells by regulating cell adhesion and invasion. Oncogene 2010, 30, 1413-1421.

109. Li, Z.; Chang, Z.; Chiao, L.J.; Kang, Y.A.; Xia, Q.; Zhu, C.; Fleming, J.B.; Evans, D.B.; Chiao, P.J. TrkBT1 induces liver metastasis of pancreatic cancer cells by sequestering Rho GDP dissociation inhibitor and promoting RhoA activation. Cancer Res. 2009, 69, 7851-7859.

110. Theodorescu, D.; Sapinoso, L.; Conaway, M.; Oxford, G.; Hampton, G.; Frierson, H. Reduced expression of metastasis suppressor RhoGDI2 is associated with decreased survival for patients with bladder cancer. Clin. Cancer. Res. 2004, 10, 3800-3806.

111. Lin, Q.; Fuji, R.N.; Yang, W.; Cerione, R.A. RhoGDI is required for Cdc42-mediated cellular transformation. Curr. Biol. 2003, 13, 1469-1479.

112. Qiu, R.G.; Chen, J.; Kirn, D.; McCormick, F.; Symons, M. An essential role for Rac in Ras transformation. Nature 1995, 374, 457-459.

113. Qiu, R.G.; Chen, J.; McCormick, F.; Symons, M. A role for Rho in Ras transformation. Proc. Natl. Acad. Sci. USA 1995, 92, 11781-11785.

114. Qiu, R.G.; Abo, A.; McCormick, F.; Symons, M. Cdc42 regulates anchorage-independent growth and is necessary for Ras transformation. Mol. Cell. Biol. 1997, 17, 3449-3458.

115. Stengel, K.R.; Zheng, Y. Essential role of Cdc42 in Ras-induced transformation revealed by gene targeting. PLoS One 2012, 7, e37317.

116. Yang, H.W.; Shin, M.G.; Lee, S.; Kim, J.R.; Park, W.S.; Cho, K.H.; Meyer, T.; Do Heo, W. Cooperative activation of PI3K by Ras and Rho family small GTPases. Mol. Cell 2012, 47, 281-290.

117. Liu, J.F.; Chevet, E.; Kebache, S.; Lemaitre, G.; Barritault, D.; Larose, L.; Crepin, M. Functional Rac-1 and Nck signaling networks are required for FGF-2-induced DNA synthesis in MCF-7 cells. Oncogene 1999, 18, 6425-6433.

118. Baranwal, S.; K Alahari, S. Rho GTPase effector functions in tumor cell invasion and metastasis. Curr. Drug Targets 2011, 12, 1194-1201.

119. Ye, D.Z.; Field, J. PAK signaling in cancer. Cell Logist. 2012, 2, 105-116. 
120. Wu, W.J.; Tu, S.; Cerione, R.A. Activated Cdc42 sequesters c-Cbl and prevents EGF receptor degradation. Cell 2003, 114, 715-725.

121. Kaneko, T.; Maeda, A.; Takefuji, M.; Aoyama, H.; Nakayama, M.; Kawabata, S.; Kawano, Y.; Iwamatsu, A.; Amano, M.; Kaibuchi, K. Rho mediates endocytosis of epidermal growth factor receptor through phosphorylation of endophilin A1 by Rho-kinase. Genes Cells 2005, 10, 973-987.

122. Ung, C.Y.; Li, H.; Ma, X.H.; Jia, J.; Li, B.W.; Low, B.C.; Chen, Y.Z. Simulation of the regulation of EGFR endocytosis and EGFR-ERK signaling by endophilin-mediated RhoA-EGFR crosstalk. FEBS Lett. 2008, 582, 2283-2290.

123. Narumiya, S.; Tanji, M.; Ishizaki, T. Rho signaling, ROCK and mDial, in transformation, metastasis and invasion. Cancer Metast. Rev. 2009, 28, 65-76.

124. Tanji, M.; Ishizaki, T.; Ebrahimi, S.; Tsuboguchi, Y.; Sukezane, T.; Akagi, T.; Frame, M.C.; Hashimoto, N.; Miyamoto, S.; Narumiya, S. mDial targets $\mathrm{v}$-Src to the cell periphery and facilitates cell transformation, tumorigenesis, and invasion. Mol. Cell. Biol. 2010, 30, 4604-4615.

125. Albiges-Rizo, C.; Destaing, O.; Fourcade, B.; Planus, E.; Block, M.R. Actin machinery and mechanosensitivity in invadopodia, podosomes and focal adhesions. J. Cell Sci. 2009, 122, 3037-3049.

126. Yamaguchi, H.; Lorenz, M.; Kempiak, S.; Sarmiento, C.; Coniglio, S.; Symons, M.; Segall, J.; Eddy, R.; Miki, H.; Takenawa, T. Molecular mechanisms of invadopodium formation the role of the N-WASP-Arp2/3 complex pathway and cofilin. J. Cell Biol. 2005, 168, 441-452.

127. Yamaguchi, H.; Condeelis, J. Regulation of the actin cytoskeleton in cancer cell migration and invasion. BBA-Mol. Cell Res. 2007, 1773, 642-652.

128. Pignatelli, J.; Tumbarello, D.A.; Schmidt, R.P.; Turner, C.E. Hic-5 promotes invadopodia formation and invasion during TGF- $\beta$-induced epithelial-mesenchymal transition. J. Cell Biol. 2012, 197, 421-437.

129. Kurisu, S.; Suetsugu, S.; Yamazaki, D.; Yamaguchi, H.; Takenawa, T. Rac-WAVE2 signaling is involved in the invasive and metastatic phenotypes of murine melanoma cells. Oncogene 2004, 24, 1309-1319.

130. Sakurai-Yageta, M.; Recchi, C.; Le Dez, G.; Sibarita, J.-B.; Daviet, L.; Camonis, J.; D'Souza-Schorey, C.; Chavrier, P. The interaction of IQGAP1 with the exocyst complex is required for tumor cell invasion downstream of Cdc42 and RhoA. J. Cell Biol. 2008, 181, 985-998.

131. Vishnubhotla, R.; Sun, S.; Huq, J.; Bulic, M.; Ramesh, A.; Guzman, G.; Cho, M.; Glover, S.C. ROCK-II mediates colon cancer invasion via regulation of MMP-2 and MMP-13 at the site of invadopodia as revealed by multiphoton imaging. Lab. Invest. 2007, 87, 1149-1158.

132. Lizárraga, F.; Poincloux, R.; Romao, M.; Montagnac, G.; Le Dez, G.; Bonne, I.; Rigaill, G.; Raposo, G.; Chavrier, P. Diaphanous-related formins are required for invadopodia formation and invasion of breast tumor cells. Cancer Res. 2009, 69, 2792-2800.

(C) 2013 by the authors; licensee MDPI, Basel, Switzerland. This article is an open access article distributed under the terms and conditions of the Creative Commons Attribution license (http://creativecommons.org/licenses/by/3.0/). 